\title{
The pattern of peri-hilar and hilar arterial branching in kidney allografts of living donors
}

\author{
Sepideh Zununi Vahed ${ }^{\mathbb{1}}$, Yaghoob Asadi $^{1}$, Farahnoosh Farnood $^{1}{ }^{\circledR}$, Maryam Alimirzaloo $^{1}$, Seyed \\ Sadroddin Rasi Hashemi ${ }^{1}$, Bahram Niknafs ${ }^{1}$, Habibeh Mohamadian ${ }^{1}$, Mohammadali Mohajel Shoja ${ }^{2}$, \\ Hamid Noshad $^{1 * \mathbb{D}}$, Mohammadreza Ardalan $^{1 * \mathbb{D}}$
}

${ }^{1}$ Kidney Research Center, Tabriz University of Medical Sciences, Tabriz, Iran

${ }^{2}$ Department of Surgery, University of Illinois at Chicago-Metropolitan Group Hospitals (UIC-MGH), Chicago, Illinois, USA

\section{A R T I C L E IN F O}

Article Type:

Original

\section{Article History:}

Received: 7 June 2020

Accepted: 4 Sep. 2020

Published online: 24 Sep. 2020

\section{Keywords:}

Renal artery

Morphology

Branching pattern

Renal transplantation

End-stage renal diseases

\begin{abstract}
A B S T R A C T
Introduction: Kidney transplantation gives us the opportunity to study kidney vascular anatomical variations and their probable effect on allograft survival.

Objectives: The aim of this study was to evaluate the renal arteries' branching patterns of the engrafted kidneys and their impact on transplant outcomes.

Patients and Methods: Three hundred patients who received kidney transplantation between 2014 -2017 were included. Peri-hilar and hilar branching patterns of the engrafted kidney were studied by reviewing the archived CT angiographies of donors and then they categorized based on the existing knowledge in this field. Clinical data were also gathered from medical records and recipients' latest clinical and laboratory evaluations.

Results: Based on peri-hilar and their corresponding hilar branching patterns, our morphology findings were classified into 17 groups. From different peri-hilar branching patterns, the fork pattern was more common which is detected in $95 \%$ (242) while the ladder pattern was observed in 5\% (13) of kidney grafts. In a later branching sub-categorization, among the fork pattern, $65.2 \%$ (158) were duplicated and 34.7\% (84) had triplicated hilar branching patterns. There was not a statistically significant correlation between each of those patterns and allograft function $(P>0.05)$.

Conclusion: Peri-hilar and hilar branching patterns of the kidney allografts' renal artery were different, but they follow certain patterns. Although it may not influence the allograft survival, it provides us precise knowledge about renal vasculature patterns and outcome of probable vascular events meanwhile; it could be useful in the field of transplantation.
\end{abstract}

Implication for health policy/practice/research/medical education:

Peri-hilar and hilar branching patterns of the engrafted kidneys were studied in recipients. The branching pattern of the renal arteries had high morphological variations; however, they follow certain patterns. The knowledge of the anatomical variations in the renal arteries is important when planning the radiologic or surgical procedures, since lack of information on their variability can lead to severe complications.

Please cite this paper as: Zununi Vahed S, Asadi Y, Farnood F, Alimirzaloo M, Rasi Hashemi SS, Niknafs B, et al. The pattern of peri-hilar and hilar arterial branching in kidney allografts of living donors. The pattern of peri-hilar and hilar arterial branching in kidney allografts of living donors. J Renal Inj Prev. 2021; 10(3): e23. doi: 10.34172/jrip.2021.23.

\section{Introduction}

Renal transplantation is the best therapeutic option for end-stage renal disease (ESRD) patients (1); however, lack of enough supply is the main limitation (2). Moreover, disparity between demands and supply is overgrowing in upcoming years (3) mainly because of increasing prevalence of ESRD. Anatomical variation of the kidney as a solid organ has a significant impact on surgical decision and transplant outcome; therefore, understanding the variations in the anatomy of the renal arteries is necessary (4).

Kidney allograft is a source of anatomical study to brush up our knowledge about the variations and branching pattern of renal arteries. These variations have been defined in the origin and number of the renal arteries and their terminal division near the hilum of the kidney (5). Understanding the anatomical pattern also has some benefits for donor safety since it is the most important issue 
of living donor organ transplant programs where specific anatomical variations prevent a potential donor to donate his or her kidney (6). Prior to kidney transplantation, in cases of living donors, we should have enough knowledge about the renal artery. The imaging studies on potential donors help us to understand the number of running arteries and their length and branching. Potentially, harvesting kidney requires careful preoperative evaluation and knowledge about the main renal artery and branching (5). Surgical complications in renal transplantation are also related to renal artery and renal vein. Anastomosis is also affected by their anatomy since it is an essential clinical issue that may increase hospital stay, procedure costs and morbidity (7). One of the controversial issues in the literature is the terminology used in the description of the anatomical variations. Moreover, there are significant differences in the classification schemes and findings of studies. These broad renal artery diversities are generally because of the various developmental positions of the kidney (8).

Renal vascular segmentation was originally recognized by John Hunter in 1794, but the first internationally accepted detailed account of the primary pattern was given by Gray in 1954 (9). However, considering these traditional models, the extra-parenchymal (peri-hilar) division and branching pattern of the renal artery are ignored. In 2008, Shoja et al described nine types of perihilar branching patterns for the morphology of main renal arteries that exhibit a high individual variability (10).

\section{Objectives}

The aim of this study was to determine the variants of the peri-hilar branching of the main renal artery and their association with graft survival. The knowledge of the anatomical variations in the renal arteries is important when planning radiologic or surgical procedures, where the lack of awareness of the variability can lead to some complications.

\section{Patients and Methods}

\section{Study design}

This retrospective study was carried out at the Kidney Research Center of Tabriz University of Medical Sciences, among 300 kidney transplant recipient who received their allograft from 2014 to 2017. All of them underwent preoperative conventional CT angiography and those with supplementary main renal arteries and early branching of main renal artery (less than one centimeter from its origin) were excluded. Based on branching pattern nomination suggested by Shoja et al (10), we classified the arterial branching patterns into two main patterns consisting of the fork type and the ladder type. In the fork type pattern, there was a common point of division while in the ladder type pattern there were the sequential branching types. Each of these patterns, then subcategorized into future subtypes. It is important to note that those with multiple or bilateral renal arteries and polar arteries were not included because they were not entered to the list of candidate donors. Clinical data including history of graft rejection or delayed graft function were extracted from medical recorded files. The function of transplanted kidney was evaluated on the basis of more recent serum creatinine $(\mathrm{sCr})$ measurement.

First year and $3^{\text {th }}$ years renal allograft status was evaluated using the following serum creatinine-based categorization: a) patients with good graft function ( $\mathrm{sCr}$ $<1.6 \mu \mathrm{mol} / \mathrm{L}$ ), b) patients with an acceptable graft function $(1.6<\mathrm{sCr}<2.5 \mu \mathrm{mol} / \mathrm{L}), \mathrm{c})$ patients with moderate graft function $(2.5<\mathrm{sCr}<4)$ and $\mathrm{d})$ patients with poor graft function $(\mathrm{sCr}>4 \mu \mathrm{mol} / \mathrm{L})$.

\section{Ethical issues}

Ethical issues were respected in accordance with the Helsinki Declaration 1975, as revised in 1983. This study was approved at ethical committee of Tabriz University of Medical Sciences (IR.TBZMED.REC.1397.199). Patients signed the informed consents. This study was extracted from the M.D thesis of Yaghoob Asadi at Tabriz University of Medical Sciences (Thesis\#59759).

\section{Data analysis}

All values were presented as the mean \pm SD or median. Statistical analyses were performed by the IBM SPSS 17.0 Software (SPSS, Inc.). Additionally, $P$ value less than 0.05 was considered statistically significant.

Results

This study was conducted on 255 living donor kidney allograft recipients who were transplanted in Imam Reza Hospital, Tabriz University of Medical Sciences, Tabriz, Iran. We excluded 45 patients who received allograft from a deceased donor during this period. The results of CT angiography images of the donated kidney in living donors revealed different peri-hilar branching patterns. On the basis of our arbitrary branching patterns, we found 19 different peri-hilar and hilar branching patterns of main renal arteries. A fork pattern was observed in 95\% $(\mathrm{n}=242)$ [56.8\% duplicated $(\mathrm{n}=145)$ and $38 \%$ triplicated $(n=97)]$ and a ladder pattern in $5 \%(n=13)$ of the kidneys (Figure 1). The main cardinal morphologies of primary and secondary peri-hilar branches of main renal artery were as follows (Figure 2);

1. Duplicated fork main artery with ladder upper and duplicated lower branches ( $\mathrm{n} ; 47,18.4 \%$ )

2. Duplicated fork main artery with duplicated upper branch and ladder lower branch (n;30, $11.8 \%)$

3. Duplicated fork of main artery with upper and lower ladder branches (n; 14, 36\%)

4. Triplicated fork main artery with ladder upper and middle and duplicated lower branch (n; 32, 12.5\%) 


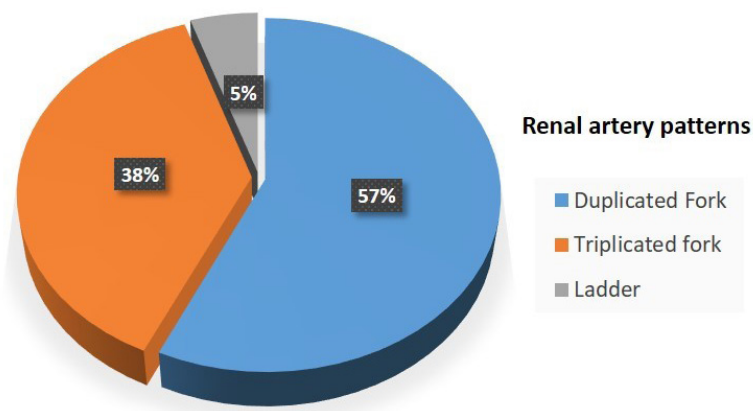

Figure 1. Branching pattern of the main renal artery.

5. Triplicated fork main artery with ladder upper, middle and lower branch ( $\mathrm{n} ; 24,9.4 \%)$

6. Triplicated fork main artery with duplicated middle and lower branch (n; 20, 7.8\%)

Other types of the branches of main renal artery were presented in Figure 2. Patient allograft function on the basis of serum creatinine level had the following results: $69 \%$ of transplanted patients $(n=177)$ were in normal graft function and their serum creatinine was below 1.6 $\mu \mathrm{mol} / \mathrm{L}, 13 \%(\mathrm{n}=32)$ had mild to moderate renal allograft dysfunction (SCr levels were between 1.7 to $2.5 \mu \mathrm{mol} / \mathrm{L}$ ), $18 \%(n=45)$ had moderate to severe renal allograft function with serum $\mathrm{Cr}$ between 2.5 to $4 \mu \mathrm{mol} / \mathrm{L}$. Moreover, one patient with ESRD (end-stage renal diseases) was waiting for renal replacement therapy (Figure 3).

From total 255 transplanted kidneys, 223 kidney recipients $(87.5 \%)$ were alive and 26 patients were died due to different etiologies including sepsis $(4.3 \%$, $\mathrm{n}=11)$, cardiovascular complications $(2.7 \%, \mathrm{n}=7)$, surgical complications $(2 \%, \mathrm{n}=5)$, graft loss $(1.2 \%, \mathrm{n}=3)$.
Condition of six patients was not evident (Figure 4). Statistically, there was no significant correlation between the pattern of graft artery and graft function and recipients survival $(P>0.05)$.

\section{Discussion}

The results of our study indicated that renal artery perihilar branching patterns did not affect the allograft survival and did not show any superiority for any specific perihilar branching pattern. Some recent studies have shown no differences in survival and complications between the allograft kidneys with different normal variant.

Awareness of the variations in renal vascular anatomy with noninvasive radiological technique is important before donor nephrectomy. This technique is useful to exclude donors with difficult anatomies. Actually all of our studied patients had only one emerging renal artery and those with polar artery or multiple aortic originated vessels were excluded and we only studied peri-hilar branching patterns (11).

CT angiography (CTA) is an excellent imaging tool for evaluation of renal vascular anatomy because it is a fast, non-invasive and highly accurate method $(12,13)$. The sensitivity of CTA for preoperative study of renal arteries reaches to $98.5 \%$ (14). The results of this study showed that peri-hilar branching is highly variable but we can categorize them within major groups. Over time, branching pattern of the renal artery has been described in many ways. The first precise classification reported by Graves and colleagues in 1954. They reported five segmental arteries, however, they did not describe the vascular differences well (9). In 1966, Fine and keen introduced three primary renal arteries (upper, lower and posterior)

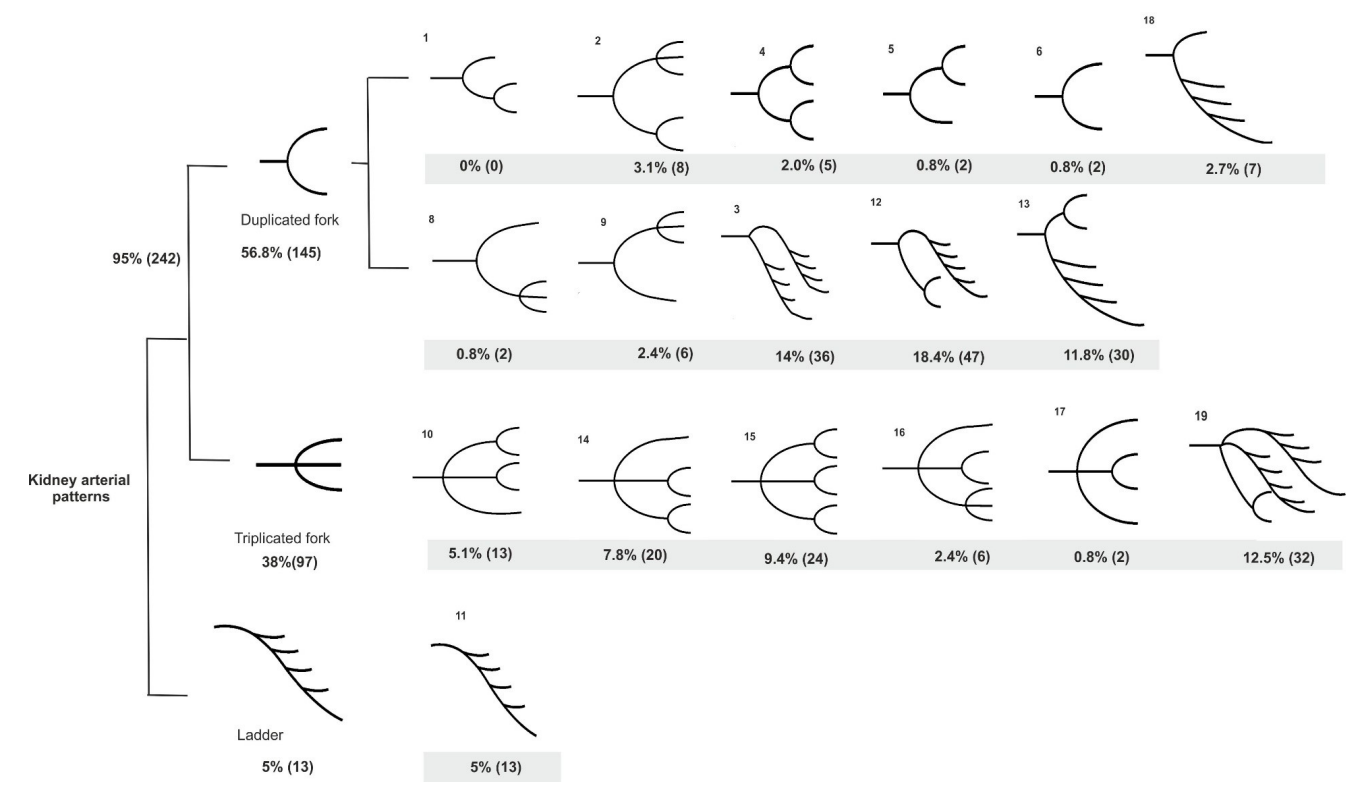

Figure 2. Renal artery branching patterns in the present study. Based on branching pattern nomination suggested by Shoja et al (10), we classified the arterial branching patterns into two main patterns consisting of the fork type and the ladder type. 


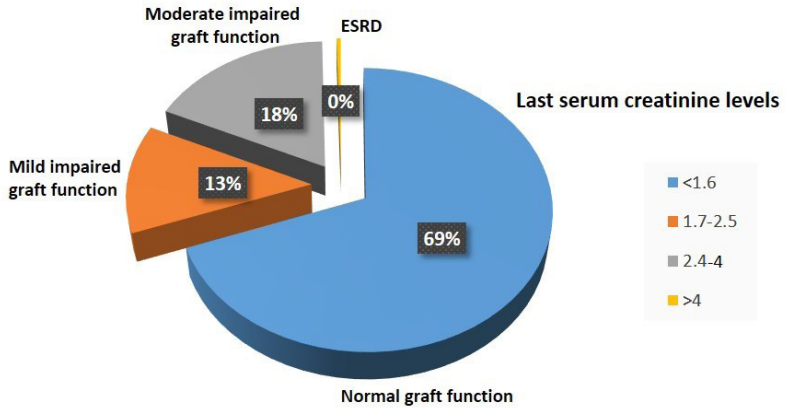

Figure 3. Serum creatinine levels in different groups of allograft recipients.

that were present in all specimens. Based on the primary artery that first arrives, they introduced four renal artery branching patterns. They also described three types of posterior arterial branching, including magistral, cruciate, and bifurcating. However, they did not distinguish such morphology for other renal artery branches (15). Cases et al (2017) classified renal artery into five types based on the renal artery origination and entering to kidney. They reported that type a, in which there is a single hilar artery entering the kidney, is the most frequent type (16). Daescu et al analyzed 60 harvested kidneys from branching point and origin of segmental arteries and represented that in $81.6 \%$ of cases, the branching of the renal artery was perihilar (17). In 2005, the anatomical variants of the segmental arteries of formalin fixed kidneys $(n=73)$ were analyzed by Weld et al. They defined a pre-segmental (pre-hilar) branching's of the renal artery (18). Shoja and colleagues performed a study on 81 donated kidney and reviewed the morphology and branching patterns of main renal artery. They employed uniform terminology and used the terms, primary, secondary and tertiary branching of the main renal artery. Additionally, they classified the perihilar branching of renal artery into two main patterns; fork and ladder and subsidiaries that emerge from different combination of those major patterns. Despite high variabilities, they found that the fork pattern with different combinations were existed in $92.6 \%$ of cases and the ladder pattern were existed in different combinations in $7.4 \%$ of cases (10). Since we performed this study in the same University hospital that Shoja et al did, we used the uniform terminology. However, patient selection criteria and imaging techniques were different between our study and theirs. In contrast to conventional angiography findings of Shoja et al, CT angiography findings of our study was just a reflection of a selected individuals who were allocated for renal donation. As we only studied the peri-hilar branching patterns of main renal artery, we can make a comparison between our results and those that are gathered from angiographic findings in unselected individuals.

In our study also the most frequent pattern was fork

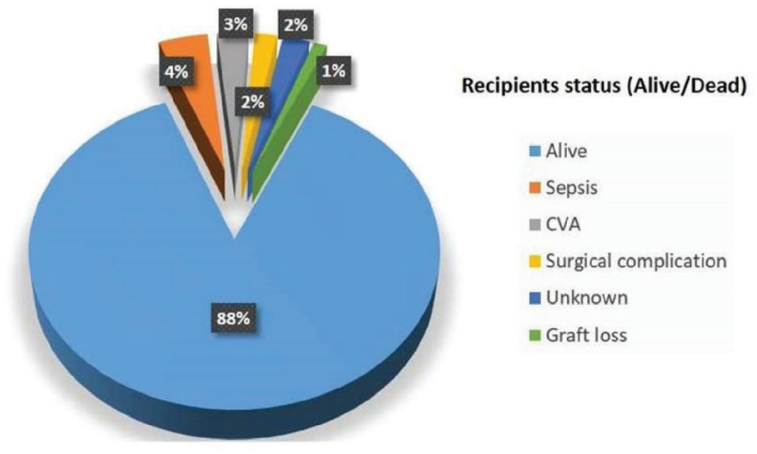

Figure 4. Recipients' status. Non-ESRD (end-stage renal diseases) related cause of death in the studied population .

pattern in different combination in 95\% (242/255) and the ladder pattern was observed in $5 \%(13 / 255)$ of the studied CT angiographic findings. In later subdivisions among the fork pattern, 65.2\% (158) had duplicated and $34.7 \%$ (84) had triplicated fork patterns. The adverse effect of multiple renal arteries on renal allograft outcome including increased risk of thrombosis, bleeding and delayed graft function has been shown in different studies.

\section{Conclusion}

Peri-hilar and hilar branching pattern of the renal arteries are characterized by high morphological variations. Our findings in connection with previous reports increase our anatomical knowledge but those patterns should not be considered as an important determiners of allograft outcome.

\section{Limitations of the study}

In our study we just did evaluate the images of CT angiography of a group of allocated donors. So, we cannot extrapolate our data to general population to estimate the incidence of accessory arteries or polar arteries because we excluded donors with multiple renal arteries, polar arteries or any other unaccepted anatomy. For these reasons we only studied the peri-hilar and hilar branching pattern in the selected individuals and it was a point of limitation in our study.

\section{Authors' contribution}

MA and MMS contributed to design of the study and revising the manuscript. YA, ML, and EA gathered the data and had interview with the kidney recipients. FF prepared the primary draft of article. SZV performed data analysis and revised the manuscript. All authors read and signed the paper manuscript.

\section{Conflicts of interest}

No conflict of interest is available.

Ethical considerations

Ethical issues including plagiarism, redundancy, and 
double publication have been completely double-checked by the authors.

\section{Funding/Support}

This manuscript was financially supported by the Kidney Research Center at Tabriz University of Medical Sciences, Iran, Tabriz (Grant \# 59759).

\section{References}

1. Kalble T, Lucan M, Nicita G, Sells R, Burgos Revilla FJ, Wiesel M. EAU guidelines on renal transplantation. Eur Urol. 2005;47:156-66. doi: 10.1016/j.eururo.2004.02.009

2. Tullius SG, Rabb H. Improving the Supply and Quality of Deceased-Donor Organs for Transplantation. N Engl J Med. 2018;378:1920-9. doi: 10.1056/NEJMra1507080

3. Liyanage T, Ninomiya T, Jha V, Neal B, Patrice HM, Okpechi I, et al. Worldwide access to treatment for end-stage kidney disease: a systematic review. Lancet. 2015;385:1975-82. doi: 10.1016/s0140-6736(14)61601-9

4. Shigueoka DC. Anatomic variations of the renal arteries, as characterized by computed tomography angiography: rule or exception? Its usefulness in surgical plannning. Radiol Bras. 2016;49:vii-viii. doi: 10.1590/0100-3984.2016.49.4e2

5. Munnusamy K, Kasirajan SP, Gurusamy K, Raghunath G, Bolshetty SL, Chakrabarti S, et al. Variations in branching pattern of renal artery in kidney donors using CT angiography. J Clin Diagn Res. 2016;10(3):AC01-3. doi: $10.7860 /$ jcdr/2016/16690.7342

6. Ghonge NP, Gadanayak S, Rajakumari V. MDCT evaluation of potential living renal donor, prior to laparoscopic donor nephrectomy: What the transplant surgeon wants to know? Indian J Radiol Imaging. 2014;24:367-78. doi: 10.4103/0971-3026.143899

7. Kang WY, Sung DJ, Park BJ, Kim MJ, Han NY, Cho SB, et al. Perihilar branching patterns of renal artery and extrarenal length of arterial branches and tumour-feeding arteries on multidetector CT angiography. Br J Radiol. 2013;86: 20120387. doi: 10.1259/bjr.20120387

8. Budhiraja V, Rastogi R, Jain V, Bankwar VJJoMS. Anatomical variations of renal artery and its clinical correlations: a cadaveric study from central India. J
Morphol Sci. 2017;30:228-233.

9. Gray's Anatomy: The Anatomical Basis of Clinical Practice. New Yor: Churchill Livingstone; 2008.

10. Shoja MM, Tubbs RS, Shakeri A, Loukas M, Ardalan MR, Khosroshahi HT, et al. Peri-hilar branching patterns and morphologies of the renal artery: a review and anatomical study. Surg Radiol Anat. 2008;30:375-82. doi: 10.1007/ s00276-008-0342-5

11. Sungura R, Mathenge I, Onyambu C. The Radiological Scrutiny of the Relation between Renal Vascular Dimensions and Anatomical Variation of Renal Arteries: The Must to Know before Renal Transplantation. J Kidney. 2017;3:4.

12. Aristotle S, Sundarapandian, Felicia C. Anatomical study of variations in the blood supply of kidneys. J Clin Diagn Res. 2013;7:1555-7. doi: 10.7860/jcdr/2013/6230.3203

13. Meng X, Mi Q, Fang S, Zhong W. Preoperative evaluation of renal artery anatomy using computed tomography angiography to guide the superselective clamping of renal arterial branches during a laparoscopic partial nephrectomy. J Exp Therap Med. 2015;10:139-144.

14. Raman SS, Pojchamarnwiputh S, Muangsomboon K, Schulam PG, Gritsch HA, Lu DS. Utility of 16-MDCT angiography for comprehensive preoperative vascular evaluation of laparoscopic renal donors. Am J Roentgenol. 2006;186:1630-8. doi: 10.2214/ajr.05.0983

15. Fine H, Keen EN. The arteries of the human kidney. J Anat. 1966;100: 881-94.

16. Cases C, Garcia-Zoghby L, Manzorro P, ValderramaCanales FJ, Munoz M, Vidal M, et al. Anatomical variations of the renal arteries: Cadaveric and radiologic study, review of the literature, and proposal of a new classification of clinical interest. Ann Anat. 2017;211:61-8. doi: 10.1016/j. aanat.2017.01.012

17. Daescu E, Zahoi DE, Motoc A, Alexa A, Baderca F, Enache A. Morphological variability of the renal artery branching pattern: a brief review and an anatomical study. Rom J Morphol Embryol. 2012;53:287-91.

18. Weld KJ, Bhayani SB, Belani J, Ames CD, Hruby G, Landman J. Extrarenal vascular anatomy of kidney: assessment of variations and their relevance to partial nephrectomy. Urology. 2005;66:985-9. doi: 10.1016/j.urology.2005.05.023

Copyright $\odot 2021$ The Author(s); Published by Nickan Research Institute. This is an open-access article distributed under the terms of the Creative Commons Attribution License (http://creativecommons.org/licenses/by/4.0), which permits unrestricted use, distribution, and reproduction in any medium, provided the original work is properly cited. 